\title{
Feuilleton.
}

\section{Der Erbe Rokitansky's über die Aufgaben und Ziele der pathologischen Anatomie.}

Prof, Dr. R. Heschl. Die pathologische Anatomie als medicinische und academische Doctrin. Antritts-Vorlesung.

\section{II.}

Selten wird es vorgekommen sein, dass ein Mann von der weitragenden Berleutung, eines Rokitansky, ohne allen äusseren Zwang, lediglich durch seine innere Ueberzengung dazu getrieben, nur um der Sache selbst willen, eine so radicale Revision seiner Arbeiten und seiner Anschauungen vornimmt, wie Rokitansky innerhalb der wenigen Jahre, die zwischen jener Vorrede, der ersten und der dritten Auflage seines Werkes liegt, die 1855 erschienen ist. Seit der Veröffentlichung seines Handbuches, sagt er selbst, "sei die pathologische Anatomie mit so vielen und so einflussreichen Thatsachen bereichert worden, dass das vorliegẹnde Lehrbuch seinem Inhalte nach wesentlich verschieden von jenem geworden. Dazu trägt ferner bei, dass ich mich bemühte, strenge an dem Factischen zu halten und das Buch zu einem Leitfaden bei meinen Vorträgen über pathologische Anatomie und beim Selbststudium derselben einzurichten. Es etscheint deshalb dasjenige, was sich aus den Factis an Schlussfolgerungen und Ansichten ergiebt, bloss seinen Grundzügen nach angedeutet. Hierin wird man übrigens herausfinden, woran ich noch zur Zeit halte und was ich aufgegeben habe." Was das Auf- gegebene anbetriff, so erweist es sich in der That als höchst umfangreich, aber was noch mehr ist, die Opfer, welche Bokitansky bringt, sind von ganz principieller Bedeutung. Grade mit denjenigen Absehnitten des allgemeinen Theiles seines Handbuches, die offenbar mit besonderer Vorliebe bearbeitet sind, geht der Verfasser am rïcksichtslosesten um. Man vergleiche nur, um Eines hervorzuheben, die siebaig Zeilen dos Abschnittes "Krankheiten des Blutes" in Bd. I. der ersten Auflage mit der entsprechenden Abtheilung der dritten. Nicht mehr ,Krankheiten", sondern "Anomalien" werden jetzt beschrieben. Die verschiedenen Krasen, auf denen das Dogma der Neuen doch zumeist beruhte, sind verschwomden, Rokitansky kennt nur noch Anomalien der Zahl und der Menge, so wie Erkrankungen der Blutkörperchen selbst. Mit Recht sagt Virchow, der auch diese Auflage kritisirt hat: „Wie viele Gelehrte mag es gehen, welche eine solche Resignation und zugleich eine solche Ausdauer besitzen, um so viel aufzugeben und nach so grossen Verlusten noch die Neigung behalten, noch weiter fortzuarbeiten! wie hoch müssen wir den Mann schätzen, der so viel vergessen und so viel lernen konnte und dem es nicht genügt, seine Irrthümer zu erkennen, sondern der die Mithe nicht scheute, sie durch neue Resultate sorgfältigerer, mehr methodischer Forschungen auch bei seinen Schülern zu verdrängen. Man mag immerhin sagen, dass jene Weichheit und Nachgiebigkeit, die wir schon oben erwähnten, diesen Fortschritt erklärlich machen, aber es bleibt em hartes Ding, die offen entfaltete Fahne einzuziehen und dem Gegner Raum zu geben, dazu gehört mehr als eine Alltagsseele! 4

Freilich konnte diese Umwandlung die Grundlagen der Persönlichkeit Rokitansky's doch nicht berühren.. War er auch von seiner hypothetischen Kraseologie zum Realismus zurückgekehrt, hatte er auch wie 
anatomisehe Iumoralpathologie bis auf ein lomöopathisches Maass zurückgebracht und die Exsudate fast noelı mehr als Virehow selbst beselıränkt "“ - aus dem Formalismus war er trotzdem noch' nicht heraus. Ja Virchow meint, dass je mehr er ,die eigentliche diehteriselie Speculation aufgegeben liabe, er inmer tiefer iı die Neigung zur morphologischen Ontologie verfallen sei. Eine gewisse Erselıeinung, die ihm mit eiıer gewissen Häufigkeit entgegentritt, gestaltet sich in seinen Gedauken allmälig zu einem Sonderwesen mit wirksamen Eigenselıften, dem er eine immer grössere Bedeutung einräumt, bis er ein anderes findet, das noch häufiger und noch mehr gesondert ist."

Mit gewohnter Schärfe wird dies durch Virchow an Rok it ansky's Lelıren von den Cysten, den Masehenwerken, den strueturloseı Blasen, den Holılkolben erwiesen. Aus dem Hohlkolben wird eine Ontologie gemacht. '„Und das ist es eben, was ieh Rokitansk y noeh jetzt vorwerfen muss. Alle diese Cysten, Blasen und Masehenwerke und Hohlkolben sind, ontologiselı betrachtet, nielts als Plırasen und Formelwerk. Die zufällige Form ist nicht das Wesen." Und weiter "die Emancipation von deın Forınalismus muss die nächste Aufgabe der Wiener Sehule und ilıres Meisters sein." Mit aller Entsehiedenheit wendet sielı der Kritiker selıliesslich gegen Rokitansky's Ansehauung über die Stase, nach diesem das wichtigste Moınent in den Entzündungsvorgängen. Virchow dagegen nennt die Stase "eiu Erbstiiek der naturhistorischen Schule, weleles in der Lehre von "ler Entzündung wesentlielı ersetzt werden rnuss dureh die Fluxion (active Congestion, Reizungs-Hyperämie) und welches nur als seeundäres Plïjnomen zugelassen werden kann. Das lässt sielı aljer nicht auf dem Wege der pathologiselıen Anatomie, sondern nur experimentell entwick chlı". Und als zweite wichtige Aufgabe überweist er dann der Wiener Schule die, „die
Gebietstlıcile der patlıologiseheı Anatonic festzıstelleı". Damit war trotz aller Unıwandlungen doelı wieder die Frage zwisehen dem patlılogischen Anatomeu und dem pathologisehen Physiologen gegehen und eonstatirt, dass man in Wien noclı immer uicht die Bedeutung der experiinentelleı Methode für die Pathologie genügend erkannt hatte, mochte man auch die durch das Mikroskop nothwendig gewordenen Reformen aunehmen. Virelıow stellt dalier wiederum die Forderung „einer strengeren und nieliteruen Methode“. „Je melır der Formalismus überwunden und die eiufacheı Gesetze des organisclien Lebens in ihre allyemeine Gültigkeit aueh für die Pathologie eingesetzt werden, je mehr jede einzelne medieiniselıe Diseiplin die möglielıst vollkommenen Untersuehungsmittel auf ihreı Gebiete anwendet, um so elıer wird auch unsere Wissenselıaft diejenige Stellung erlangen, welehı ilır mit Reelıt zukoınınt. Dass aber gerade Deutsehland der Boden ist, auf dem diese Regeneration der Medicin in ilıren wesentliehsten Zweigen sieh vollenden wird, das ist die stolze Hoffnung, welelıe wir von jelıer festgelialten haben und dass die Wiener Selıule, welche sehou jetzt eimen so grossen Antheil an dieser Regeneration gelıommen liat, aueh fernerhin der Falıne des Fortselirittes treu bleiben werde, das können wir un so sicherer erwarten, wenn wir hier wiederum sehen, wie entwickelungsfällig sie ist." Irren wir uieht, so lıatte diese Entwickelungsfihigkeit dosh ilıre Grenzen und daran lag es, nicht etwa an dem Marngel guten Willens, wenn Virehow's Hoffnung bezuiglielı der durelı Rokitansky repräsentirten Wiener Selıule nicht in Erfitllung ging. Je melır Letzterer selbst sich mit den neuen Doetrinen befreutete, je opferwilliger er das über Bord warf, was zu der neuen Position nicht passte, je eifriger er sich die Resultate einer Forschung zu eigen zu unachen suchte. deren Metlode von seiner frülıeren so weit 
ablag, je energischer er in dieser Richtung selbst thätig war, um so weiter entfernte er sich von dem, was ihn eigentlich gross gemacht hat. Die beiden Bände der speciellen pathologischen Anatomie der ersten Auflage mit ihren klassischen Krankheitsbildern, werden für alle Zeiten dauern, zu der Lieblingslectüre strebender Aerzte gehören und stehen unseres Erachtens der späteren Umarbeitung weit voran. Auch Virchow sieht hierin die starke Seite Rokitansky's, „dureh die er in der Geschichte der Wissenschaft durch alle Zeiten den ehrenvollsten Platz behaupten wird. Es ist dies weder die klinische noch die chemische, noch die genetische, sondern gerade die descriptive. Rokitansky ist genau genommen der erste, wahre descriptive, pathologische Anatom und seine Schilderungen. der krankhaften Veränderungen werden für lange Zeiten als Muster betrachtet werden können. Durch ihn hat die Wissenschaft allgemein verständliche und zugängliche Typen bekommen, welche jeder leicht wieder auffindet und welche der klinischen und physiologischen Forschung als feste Anhaltpunkte dienen können. Darum ist er für die Krankheitslehre Gewissermaassen das geworden, was Linné für die Pflanzenkunde war; und darum musste man immer so himmelweit unterscheiden zwischen seiner speciellen (topographischen) pathologischen Anatomie und dem allgemeinen Theile".

Auch für die dritte Auflage wird dies vollkommen anerkannt, ja zugegehen, dass er auch in der Histologie nahezu auf den Punkt gekommen sei, der in der gröberen Anatomie seine Grösse begründet habe. "So klar wie seine Abbildungen sind auch seine Worte und auch hier versteht er es, die Sprache seinem Zweck gemäss zu bilden, manchmal etwas gewaltsam, aber doch fast immer mit einem Geschick, welches am Ende auch die Gewalt vergessen macht."
.Es ist charakteristisch und dabei doch sehr erklärlich, dass Rokitansky's Begabung für die descriptive Anatomie gerade seine Vorliebe für die Speculation in ihm hervorrief. Die Bedürnnisse des Geistes verlangen ebenso stürmisch ihre Befriedigung wie die des Körpers. Der grosse pathologische Anatom hatte allerdings die Speculation mit seltener Selbstverläugnung als für die Pathologie unzulaissig erkannt, er wendete sich jetzt aber der Philosophie selbst zu und zwar der Arthur Schopenha u er's, deren bestrickenden Reiz so Viele an sich erfahren haben. Nicht die anatomische Forschung ferner hat ihn, wie wir glauben, zum Darwinismus gefïhrt, sondern dass derselhe ebenfalls getränkt ist mit dem Blute der Naturphilosophie und ihm ein aprioristisches Postulat, welches er längst in sich trug, auf anscheinend exactwissenschaftlichen Wegen zu lösen schien. Aus dieser Verbindung der Schopenhauer'schen Philosophic mit den Anschauungen des Darwinismus ist eine eigenthümliehe Combination bei Rokitansky hervorgegangen, der er den prägnantesten Ausdruck in der kleinen Schrift , die Solidarität alles Thierlebens " verliehen hat. „Das thierische Protoplasma ursprünglich als solches verstanden, oder aus dem pflanzlichen hervorgegangen ins Dasein getreten, erhält sich in dem umgebenden Medium vermöge der seinem Hunger zur Seite gehenden Subactions- und Assimilationskraft, wächst heran, vermehrt sich auf die einfachste Weise durch Theilung, Sprossung und Ablösung, Zerfall zu winzigen, das ganze ungetheilte Leben der Urmasse enthaltenden Partikeln (Jäger) und eröflnet die Thiersehöpfung. Dieses protoplasmatische Leben beherrseht, wie sich die heutige Wissenschaft auszusprechen in der Lage ist, die gesammte Thierwelt, ihre Entwickelung, ihren Bestand in den so mannigfaltigen Formen; diese selhst, Gesundheit und Krankheit, sind Functionen des lebendigen Protoplasma," 
Rokil an sky ist es unzweifelhaft, dass „diese Welterscheinung das Gebilde des Vorstellenden, seine Vorstellung, somil eine individuelle Sehöplung jsi "“ und dadurch kommt er zu der "ernüchternden Wahrnelımıng", dass "wic wir die Thiere, auch sie - miltelst Verrichtungen, welche die Vorausselzung aller anschaulichen Erkenutniss sind - uns erkenuen, uns vorstellen, dass wir einen Bestandheil der von ihnen vorgestellten Welt ausmachıen, dass wir demnach von so vielen Thieren unzälılige Miale vorgestellt werden und consequenterweise mit dem Untergange jedes derselben in der ihm zukommenden Erseheimungsform mutergehen". Anch die pessimislische Auffassung des frankfifurter llenkers ist die seine. ,Es kanı keine Frage sein für den Unbefangenen, dass die Leiden und Loose der Thierwelt, zumal der Menschenwell, weil iiherwiegen iiber dis Freuden". "In diesen gemeinschafllichen Grunde wurzelt ihm alles, was, wenn es die Materic zur Empfindung und Intelligenz gebracht, als Emplundenes und Verstandenes, d. i. als Leiden an das Thier herantrill und ist ihm wie dieses ein unalbweisliches, der Thierwelt solidarisch zukommendes Erbe. Ja ,"lie Wurzeh alles Thicrlebens und Thierverkelirs reiclien ilın von den höchsten Kreisen heral, in das protoplasmatische Urthię"“. So Rokitansky in den letzlen Jahren scines Simmens unl Strebens!

Wir habën lange bei dem "M orgagni unseres Jahrhınderts" verweilen und ihn auf den weiten Wege begleiten miissen, der ilın vom Sectionslische des Wiener Krankenhauses an Schopenhauer und Darwin, zu der Speculation von der Solidarital alles Thierlehens fülorte. Als or vor Kurzem den Schauplatz seiner Thäligkeil verliess, un den $\Lambda$ bend des Lebens noch ganz für sich selbst zu gewimuen, erregte die Frage, wer ihn ersetzen werde, mit Rechı ein nichı gewölntiches Interesse. Dass deı' begalıteste Schüler Virchow's fast an erster Stelle genaunt wirde, war dafür charakteristisch, dass eine Wiener Sclute in der pathologiselıen Anatomie kitnm noch existirte. Eigentliche Schuiler und Jünger, die ihn auf seinem wissenschaftlichem Specialgebiete nachgeeifert liälten, hat Rokitansky ja iilıerlanpt nicht viele gebildet, denn Sıricker, dem er selbst die experimentelle Pathologie znwies, gehı von Brücke aus, jedoch Klob, Dlauby, Ileschl mögen geenannt werden. Die Verhandlungen unit dem Strasshurger Pathologen waren vergeblich. Am 12. October 1875, genau Ireissig Jialtre, „lass er als Studirender der Medicin Init seinen Collegen zum ersten Mal die Räune betrat, in denen damals die pathologische Anatomie grclelirt wurle", sprach Heschl als Nachfoloer seines

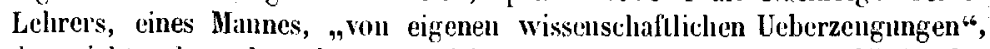
den nicht "das sehwankende Schifflein vou der schaukelnden Woge der Tagesmeinng, bald hier bald dorthiu bewegl", der niemals lediglich bestrebl war, "sieh sellsst hübsch inf der Oberfliehe zu hialten. lis war der Steuermann, der festen Blickes mil gewaltiger liraft sein Ziel verfolgte, ein Forscher ersten Ranges: Rokitanslicy". Welehe Stellung der Erbe des so Gefeierten diesenu wie der bathologisehen Pliysiologie gegenülor cimnimm, welche Anschaunngen, welche Grundsätze die seinen sind, auf welche Wuge er seine Schüler weisen will, hat Heschl mil zienlicher Beslimmtheit damals sofort ausgesprochen, wenn auch nicht immer allzuklis. Wir werden versuchen, ihn zu folgen. 